\title{
Face à la sécularisation de l'enseignement primaire, une analyse quantitative des capacités de résistance des diocèses français
}

\section{Gérard Cholvy}

\section{(2) OpenEdition \\ Journals \\ Édition électronique \\ URL : http://journals.openedition.org/trema/1846 \\ DOI : $10.4000 /$ trema. 1846 \\ ISSN : 2107-0997 \\ Éditeur \\ Faculté d'Éducation de l'université de Montpellier}

\section{Édition imprimée}

Date de publication : 1 décembre 1997

Pagination : 17 - 34

ISSN : 1167-315X

\section{Référence électronique}

Gérard Cholvy, « Face à la sécularisation de l'enseignement primaire, une analyse quantitative des capacités de résistance des diocèses français », Tréma [En ligne], 12-13 | 1997, mis en ligne le 01 décembre 1997, consulté le 19 avril 2019. URL : http://journals.openedition.org/trema/1846 ; DOI : 10.4000/trema. 1846

Ce document a été généré automatiquement le 19 avril 2019.

Trema 


\title{
Face à la sécularisation de
}

\section{l'enseignement primaire, une analyse quantitative des capacités de résistance des diocèses français}

\author{
Gérard Cholvy
}

1 Au seuil des années 1880 , alors que les républicains viennent de prendre en charge les rouages essentiels de l'État (assemblées, gouvernement, présidence de la République) deux conceptions de la société s'affrontent à propos de l'école. Pour les catholiques, la religion passe avant l'instruction, le rôle premier de l'éducation est de former des chrétiens. Les lois Guizot (1833) et Falloux (1850) avaient fait droit à cette requête en plaçant l'instruction religieuse en tête des matières enseignées à l'école élémentaire. L'instituteur fait apprendre le catéchisme diocésain, des prières sont dites en classe et le maître accompagne les enfants à l'église ou au temple. Dans la pratique, les relations entre le clergé et les instituteurs laïcs allaient de la subordination à la collaboration. Elles s'étaient dégradées à partir de 1860, en partie en raison de la concurrence opposant les maîtres congréganistes, fréquemment en charge des écoles communales et les maîtres laïcs; en partie en raison de la mentalité dominante dans la commune, l'instituteur dépendant aussi du maire. Ces raisons-là pesaient plus sans doute que le conflit idéologique entre l'Église et la société moderne, même si celui-ci est mis en avant dans beaucoup de sources écrites.

2 Ministre de l'Instruction publique depuis le 4 février 1879, Jules Ferry, comme la plupart des républicains qui sont positivistes sinon même scientistes, fonde la promotion de l'homme sur la science et oppose la raison à la révélation. «L'instruction publique, qui est le premier des services publics, doit tôt ou tard être sécularisée comme l'ont été depuis 1789 et le gouvernement, et les institutions et les lois » (discours au Sénat le 10 juin 1881).

3 Mais, condamnée par les catholiques, cette sécularisation ne peut se faire que sans eux et contre eux. En septembre 1879, le ministre a effectué une tournée dans le Midi. Il a fait le 
récit de son arrivée à Perpignan, la nuit tombée «une marche triomphale à travers le flot délirant de 20000 enthousiastes, de 20000 fanatiques des lois Ferry». A Montpellier "le débordement continue. Hier il a fallu, chemin faisant, haranguer à Béziers une foule immense... Malheur à ceux qui n'entendent pas! $\rrbracket^{1}$.

Il n'est pas question ici de retracer les péripéties de la lutte ${ }^{2}$ mais, faisant appel à la meilleure connaissance que nous commençons à avoir de la diversité française ${ }^{3} d^{\prime}$ 'évaluer les capacités de résistance des différents diocèses métropolitains, ceci à partir de quelques indices quantitatifs. Ceci fait, et à partir des effectifs scolarisés en 1893, nous esquisserons un commentaire que légitime rétrospectivement cet extrait de lettre de $\mathrm{M}^{\mathrm{gr}}$ Isoard, évêque de Chambéry : «En somme, j'ai suivi les indications fournies par le tempérament du diocèse $»^{4}$.

\section{L'évaluation des capacités de résistance}

5 Un critère simple de mesure ne serait-il pas celui des élections? Antoine Prost a montré combien le vocabulaire des professions de foi électorales était révélateur. Dans l'échantillon qu'il a choisi pour 1881, Dieu apparaît dix fois à droite, jamais à gauche; la famille 17 fois contre 0 ; à l'inverse le progrès 22 fois à gauche contre 8 à droite ${ }^{5}$. Mais les élections législatives de 1881 sont intervenues dans la précipitation, leur taux d'abstention, $31,4 \%$, est le plus élevé de l'histoire électorale de la III ${ }^{\mathrm{e}}$ République. Dans nombre de départements « les droites n'ont pas affronté la lutte ». En 1885, par contre l'Union conservatrice a tiré profit de l'impopularité de Ferry, mais celle-ci est moins due peut-être à sa politique de laïcisation qu'à ses entreprises coloniales. Retenons seulement de ces élections l'ordre suivant d'importance décroissante de la droite pour quelques départements proches:

- très forte influence : Aveyron, Lozère, Ardèche ;

- forte influence : Haute-Loire, Gard, Tarn, Haute-Garonne ;

- faible influence : Hérault, Aude, Ariège ;

- très faible influence : Pyrénées-Orientales.

Déjà, les impressions fugitives recueillies par Ferry en 1879 n'étonnent plus : la question religieuse constitue alors, et pour des décennies, la ligne de partage essentielle de l'opinion. Et plus encore au sein des populations rurales partout où l'industrialisation est réduite.

7 Nous avons donc fait appel à 7 indices de mesure, le choix étant en partie dicté par les sources disponibles. Chaque indice est affecté d'une échelle dont le 0 équivaut au point le plus bas de la résistance supposée. Le premier des indices est la pratique pascale des deux sexes, le test principal de la fidélité catholique aux yeux du clergé de l'époque. Elle suppose, en effet, la confession annuelle. L'échelle adoptée est la suivante : 


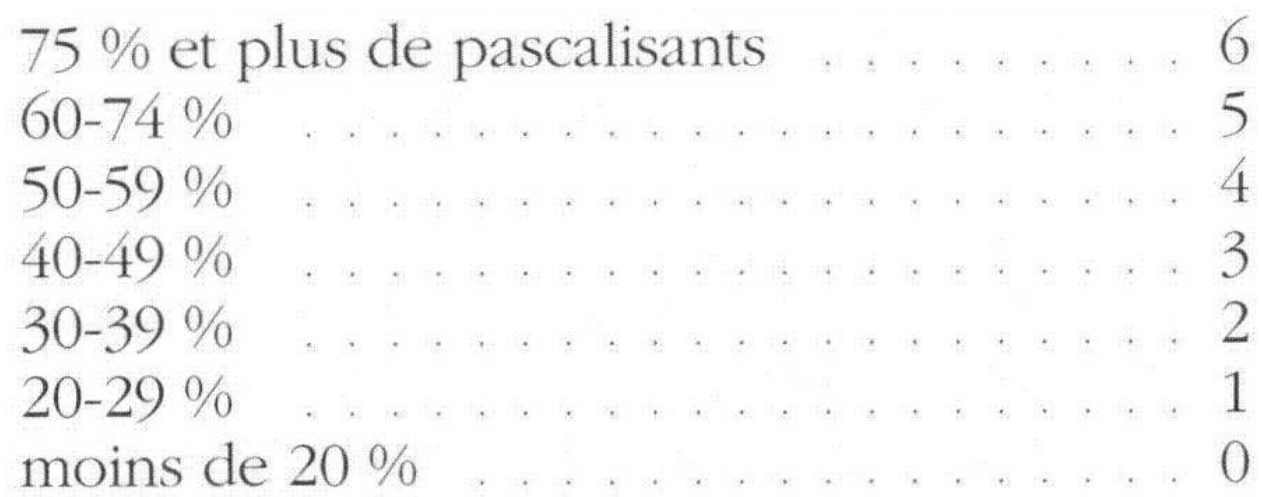

8 Assurément, il serait souhaitable d'affiner en ne retenant que la pratique des hommes et jeunes gens (13 ans et plus). Mais cela n'est pas possible sur l'ensemble de la France faute de données disponibles. L'exemple-type de la« chrétienté » est alors le diocèse de Mende avec 94 \% d'hommes à Pâques en 1910 (et 97 \% de femmes) : les rares non-conformistes sont des fonctionnaires ou des étrangers au pays. Encore est-il certain qu'une grosse majorité des instituteurs laïcs des années 1880 est pratiquante. La Haute-Loire se situe sensiblement au dessous en raison du clivage existant entre les arrondissements du Puy et d'Yssingeaux d'une part, et celui de Brioude qui est plus détaché. Dans sa moyenne, ce diocèse est comparable à celui de Rodez où $72 \%$ des hommes et $90 \%$ des femmes communient à Pâques (1876-1890). Ici également les nuances sont mieux perceptibles qu'en Lozère. Villefranche-de-Rouergue n'a que $40 \%$ d'hommes, alors qu'une cité industrielle comme Millau en compte $48 \%$. L'autre zone de faiblesse concerne le bassin minier de Decazeville. Partout ailleurs, dans les paroisses rurales, la pratique ne souffre que de rares exceptions et souvent aucune pour les femmes. L'Ardèche est plus divisée, le nord, le plateau et la montagne étant tout à fait comparables aux cantons unanimes de la Lozère. Les diocèses d'Albi, de Nîmes et de Montauban sont dans une situation intermédiaire. Dans le Tarn, en 1901, 48 \% des hommes et $85 \%$ des femmes pascalisent, la ferveur domine la montagne: il y a $57 \%$ de pascalisants dans la cité industrielle de Mazamet. Dans le Gard, grands sont les contrastes entre la partie montagneuse, où la pratique masculine est le plus souvent majoritaire et le littoral ou les tords du Rhône. La zone déprimée de cet ensemble régional se situe toutefois dans les diocèses de Montpellier (19 à $20 \%$ des hommes en 1876, $60 \%$ des femmes), Carcassonne (17 \% et $57 \%$ en 1885), Perpignan (13,5 et $57 \%$ en 1887) et Pamiers (19\% et $56 \%$ en 1912). Encore s'agitil de moyennes. Dans l'Hérault, il existe quelques cantons très pratiquants (La Salvetat, Ganges) alors que les campagnes du Biterrois et du Minervois sont dominées par un conformisme anticlérical masculin. Dans l'Aude, le même contraste existe entre le Narbonnais et les Corbières d'une part ( $5 \%$ d'hommes) et le Lauragais (35 à $55 \%$ d'hommes).

9 Le second indice retenu concerne les élèves (garçons et filles) qui ont un maître congréganiste (frère ou religieuse) dans l'enseignement public ou libre en $1880^{7}$ : 
Valeur indicielle de résistance à la laïcisation

$60 \%$ et plus

$50-59 \%$

$40-49 \%$

$30-39 \%$

$20-29 \%$

moins de $20 \%$

Le troisième indice est la proportion des protestants pour 10000 habitants. Ceci part de l'hypothèse selon laquelle l'émulation joue lorsqu'un protestantisme fervent côtoie des communautés catholiques (cf. tableau ci-dessous) :

$\begin{array}{lccc}\text { Départements } & \text { Population en 1881 } & \text { Protestants } & \% \\ \text { Gard } & 423000 & 120000 & 28,4 \\ \text { Lozère } & 143000 & 20000 & 14,0 \\ \text { Ardèche } & 384000 & 48000 & 12,5 \\ \text { Tarn } & 359000 & 17000 & 4,7 \\ \text { Hérault } & 430000 & 14000 & 4,3 \\ \text { Haute-Loire } & 313000 & 8500 & 2,7 \\ \text { Aveyron } & 415000 & 3000 & 0,7\end{array}$

Valeur indicielle de résistance à la laïcisation

Plus de $1000 / 10000$ hab. ....... 5

401 à $1000 / 10000$ hab. ........... 4

151 à $400 / 10000$ hab. ............ 3

51 à $150 / 10000$ hab. ............. 2

5 à $50 / 10000$ hab. ................ 1

moins de $5 / 10000$ hab. ........... 0

11 Le quatrième indice est celui des pétitions défendant la loi de 1875 sur la liberté de

l'enseignement supérieur. Ferry voulant revenir sur l'existence des jurys mixtes, une pétition circula en 1879 qui recueillit 1775000 signatures. Il n'est pas sans intérêt de mesurer à cette occasion la mobilisation des populations. Dans les calculs, la population protestante a été déduite lorsqu'elle est supérieure à $5 \%$. On pensera peut-être que cette question intéressait fort peu les populations rurales. Or... c'est la Lozère qui, avec 162 pétitions par 10000 habitants, contre 48 de moyenne nationale, distance tous les départements français avec 23200 signatures (contre 9087 dans l'Hérault) : on mesure ici l'ascendant d'un clergé-paysan sur un peuple de ruraux en grande partie paysans : ${ }^{8}$ 


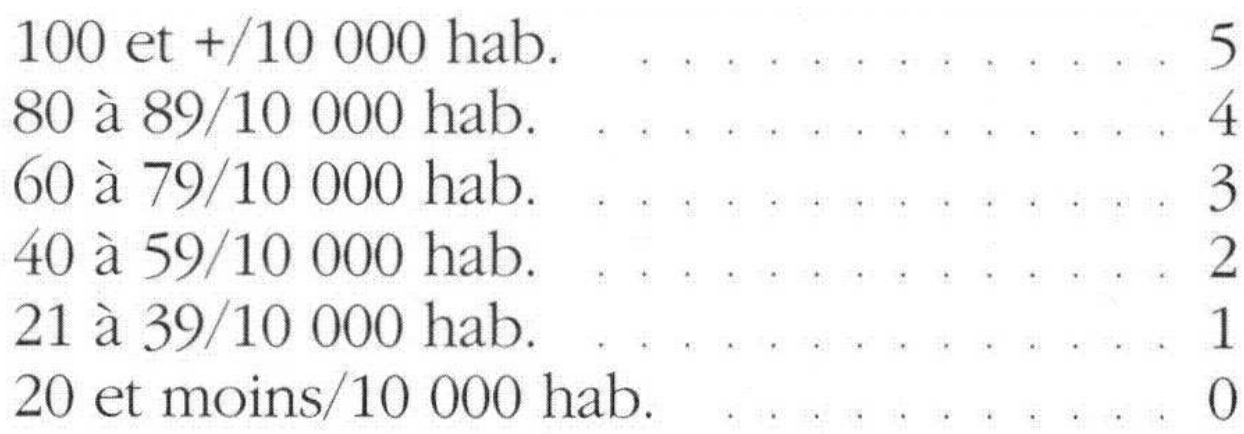

Le cinquième indice est politique. Il résulte du scrutin du 31 mai 1887 sur l'ordre du jour Barodet : ce radical intransigeant se heurta à une coalition de conservateurs et de républicains modérés: la géographie de ce scrutin annonce les élections du $\mathrm{XX}^{\mathrm{e}}$ siècle avec le passage à droite de la France de l'Est: classement de 0 (aucun élu hostile au radicalisme) à 7 (tous les élus hostiles).

Le sixième indice est celui des lecteurs de La Croix en 1893. On a là une représentation des minorités actives au sein des pratiquants, ceux à qui on va commencer à donner le nom de « militants »:

Valeur indicielle de résistance à la laïcisation

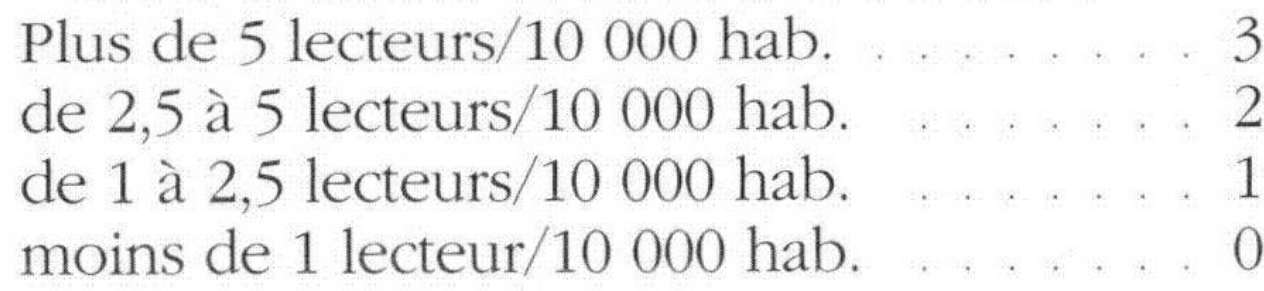

Enfin, c'est le recrutement sacerdotal dans la première génération ayant pu connaître l'enseignement primaire laïcisé qui est pris en compte : elle arrive à l'âge de l'ordination (25 à 29 ans) en 1900-1904. Le taux de vocation a été calculé protestants déduits. Il ne concerne que le clergé diocésain car le nombre des vocations dans les ordres religieux, soit comme prêtres soit comme frères est impossible à connaître sauf dans le cas d'études particulières : c'est le cas en Lozère ou dans l'Aveyron, toutefois, où il est, lui aussi, fort important. Dans les pays pauvres et là où les familles sont nombreuses, le sacerdoce est, ou plus exactement, peut être, un vecteur de promotion sociale. Mais à partir des lois laïques il en est de même pour la carrière d'instituteurs celle-ci bénéficiant, de surcroît, des faveurs gouvernementales. Les comparaisons seraient donc très souhaitables entre l'un et l'autre recrutement. On n'oubliera pas, car c'est très important, que dans les chrétientés, la vocation enseignante se traduit, chez les hommes, par l'importance du recrutement des frères enseignants, frères des Écoles chrétiennes, mais aussi frères de l'Instruction chrétienne (Ploërmel) petits frères maristes, clercs de SaintViateur, frères du Sacré-Cour, frères de la Sainte-Famille, ces derniers instituts, nés au $\mathrm{XIX}^{\mathrm{e}}$ siècle, correspondant à l'essor de la scolarisation dans les zones rurales, là où le maître a longtemps été unique : les «grands »frères, autrement dit les Frères des Écoles chrétiennes, n'ouvraient une école qu'au nombre de trois au minimum. Les autres instituts envoient leurs frères deux par deux et sont davantage polyvalents. 
La totalisation des colonnes 1 à 7, (cf. tableaux, pp. 25 et 26), permet un classement en 6 régions bien distinctes, l'écart entre les diocèses étant de 1 à 33 (colonne 8).

1. Capacité de résistance jugée le plus souvent très faible dans une grande zone prenant en écharpe la France, (cf. carte, p. 23), du Bassin parisien au Centre et au littoral atlantique, des Charentes aux Landes. On ne s'étonne pas d'y trouver l'Yonne qui est le pays de Paul Bert, ministre de l'Instruction publique et, pour qui le clergé était «le phylloxéra de nos paroisses ». Dans les Charentes, les protestants sont aussi détachés du culte que les catholiques. Le Limousin fait partie de cette aire de faiblesse qui culmine dans la Creuse qui est à l'indice 1, la Creuse l'exemple-type des pays ruraux « déchristianisés ».

2. Vient ensuite le littoral méditerranéen, ensemble également déprimé, et particulièrement le Var (3) et les Pyrénées-Orientales (4) mais, on le sait, moins homogène en raison des contrastes qui existent d'un canton à l'autre, sinon souvent d'une commune à l'autre : le bon exemple étant l'Hérault (10).

3. Dans le Nord-Pas-de-Calais (16-17) il existe des points d'appui pour une résistance: qu'il suffise de penser à la plaine flamande et à la région de Boulogne.

4. L'Est est pratiquant et plus encore si l'on tient compte des départements annexés qui figurent pour mémoire sur la carte.

5. L'Ouest armoricain auquel se rattache la Seine-Maritime, constitue un bloc de résistance virtuelle ne comptant alors que peu d'exceptions.

6. Enfin, du Pays basque à la Savoie, les diocèses de montagne ou mixtes constituent des terres aux capacités de résistance virtuellement considérable. Il faut noter que les indices de 27 pour l'Aveyron, 29 pour la Lozère et 33 pour l'Ardèche, dépassent ceux de l'Ouest : Ille-etVilaine, Côtes-du-Nord et Vendée 24, Morbihan 25.

Dans une lettre à l'un de ses prêtres, $\mathrm{M}^{\mathrm{gr}}$ de Cabrières, évêque de Montpellier, disait que "les évêques avaient derrière eux toute l'armée du peuple chrétien " (25 avril 1879). Ceci semble objectivement vrai mais dans une trentaine de diocèses seulement. C'est contestable ailleurs... à commencer par le diocèse de Montpellier où la pratique pascale masculine dans certains cantons était inférieure à $6 \%$. L'évêque a du mal à se départir de ses réflexes de Nîmois. Il compte beaucoup d'autre part sur les femmes, nettement plus pratiquantes dans le Midi, et sur les nombreux notables de la ville épiscopale.

\section{Une résistance efficace?}

17 Les débats parlementaires qui ont précédé le vote de la loi du 28 mars 1882, les problèmes suscités par son application, ont eu une intense répercussion dans l'ensemble du pays. En beaucoup de communes rurales, en particulier, la question scolaire est la pierre de touche des clivages politiques jusqu'à la loi Debré de 1959. Ferry qui était partisan d'une morale sans épithète "la bonne vieille morale de nos pères, la nôtre, la vôtre, car nous n'en avons qu'une» (10 juin 1881 au Sénat), justifiait l'article premier du texte de la loi «l'instruction religieuse sera donnée en dehors des heures de classe... par les ministres des différents cultes, conformément au vœu exprimé par les familles »en invoquant la liberté de conscience des instituteurs. L'argument prêtait à discussion comme le montre l'usage dans les départements qui sont restés concordataires : de nombreux instituteurs laïcs n'auraient $\mathrm{vu}$, alors, aucune objection à continuer de donner l'enseignement religieux. On sait aussi que les devoirs envers Dieu furent maintenus dans les programmes (2 août 1882) et qu'ils constituent le dernier chapitre des manuels de morale. On se souvient moins des hésitations du ministre: en 1880 il envisageait d'autoriser les ministres des cultes « ̀̀ 
donner l'instruction religieuse dans les locaux scolaires »après avis du Conseil municipal (Journal officiel du 22 décembre 1880). Plusieurs Consistoires protestants étaient favorables à cette disposition. Mais Ferry dut compter avec la surenchère radicale et celle des députés groupés autour de Gambetta. Lors du scrutin du 23 décembre 1880, l'extrêmegauche mêla ses voix à celles de la droite pour repousser, par 220 voix contre 200, l'article 1 prévoyant la venue du ministre des cultes à l'école. Des sénateurs catholiques qui refusaient la politique du pire reprirent cette disposition sous forme d'amendement. Mais Ferry, sachant que la Chambre ne suivra pas le Sénat désavoua ses propres paroles. La loi du 28 mars 1882 ne fut votée qu'après un renouvellement partiel du Sénat. Elle consacrait éventuellement le jeudi à l'enseignement religieux mais en dehors de l'école. La totale victoire d'un camp ne préfigurait pas l'apaisement.

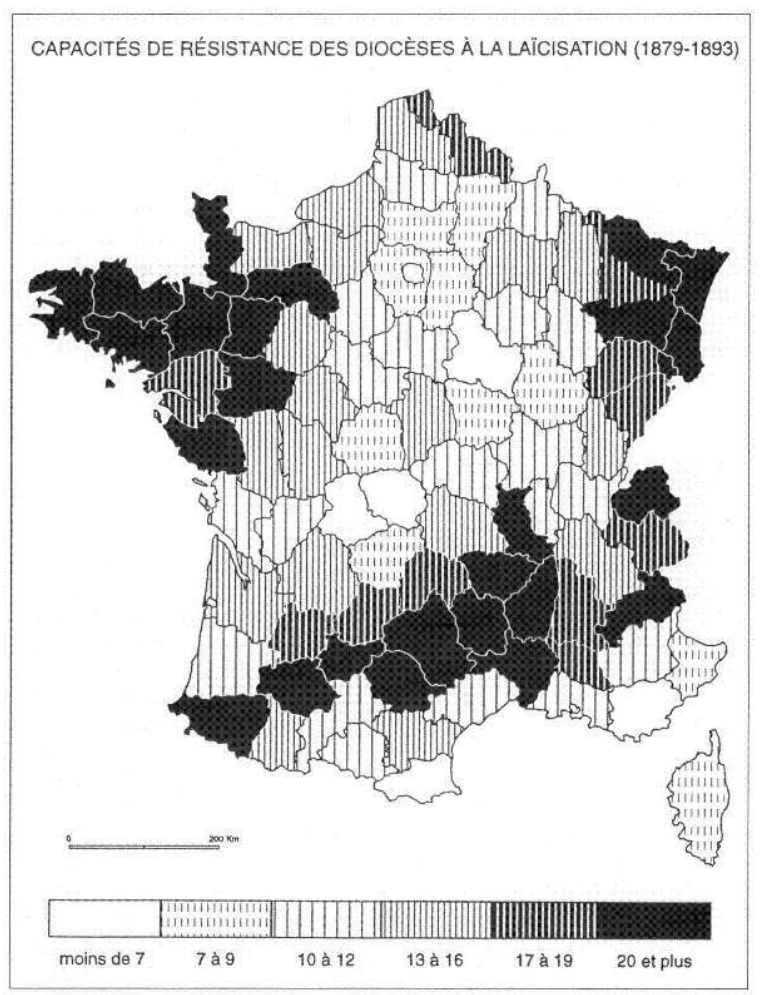

Dix ans après le vote de la loi, l'enseignement primaire congréganiste (communal ou libre) a régressé de $36,4 \%$ à $29,9 \%$ des élèves, ceci étant une moyenne nationale. La perte était plus sensible pour les garçons $(-11,7 \%)$ que pour les filles $(-8,6 \%)$. Il y a deux explications à cela : l'attachement aux religieuses, qui étaient souvent aussi gardesmalades, était plus grand et bien des républicains en tenaient compte y compris dans leur propre famille. La seconde raison tient à la loi Goblet de 1886 laïcisant le personnel des écoles communales. Elle n'accordait qu'un délai de cinq ans pour les frères alors que les religieuses en fonction pouvaient y demeurer jusqu'à leur retraite. Restait encore la question des bâtiments scolaires qui résultaient parfois d'une donation assortie de la condition expresse de la présence de frères ou de religieuses. Aussi existait-il encore en 189232249 garçons dans les écoles publiques congréganistes et surtout 460557 filles. Mais la répartition des pertes de l'école congréganiste révèle de fortes disparités. Ainsi les 7 départements armoricains ont-ils gagné 36000 élèves; la Seine, la Seine-et-Oise et la Seine-et-Marne, 42500 ; la Meurthe-et-Moselle 4700 ; la Marne et la pauvre et petite Lozère 1000 chacun... 
Dans quelle mesure ont joué les capacités de résistance prêtée aux diocèses ? La colonne 9 concerne les effectifs des écoles congréganistes en 1892-1893 :

Valeur indicielle de résistance à la laïcisation

- forte chute des effectifs et du pourcentage

- chute des effectifs égale ou inférieure à $15 \%$ mais forte chute du pourcentage .....................

- chute des effectifs mais faible variation du pourcentage _... 2

- effectif stable mais chute du pourcentage . . . . . . . . . . 3

- effectif stable et faible variation du pourcentage ........ 4

- hausse des effectifs mais chute du pourcentage ....... . . 5 5

- hausse des effectifs et stabilité du pourcentage ........ 6

- hausse des effectifs et hausse du pourcentage ......... . . 7

\begin{tabular}{l|c|c|c|c|c}
\hline Diocèses & Total & \multicolumn{2}{|c|}{$\begin{array}{c}\text { Résistance à la chute des } \\
\text { effectifs et du \% }\end{array}$} & \multicolumn{2}{c}{$\begin{array}{c}\text { Effectifs en hausse } \\
\text { (catégories 5, 6, et 7) }\end{array}$} \\
\hline Résistants & 37 & 20 & 51,3 & 10 & $25,6 \%$ \\
\hline Affaiblis & 48 & 19 & 39,6 & 7 & $14,6 \%$ \\
\hline
\end{tabular}

La moitié des diocèses ayant des capacités de résistance ont effectivement résisté ; un quart a pu accroître ses effectifs congréganistes, soit au total $76,9 \%$ ayant tenu ou consolidé la situation initiale. Dans les départements offrant au départ des conditions défavorables il y a quand même eu une résistance voire pour 7 d'entre eux des effectifs en hausse.

Valeurs indicielles de résistance à la laïcisation

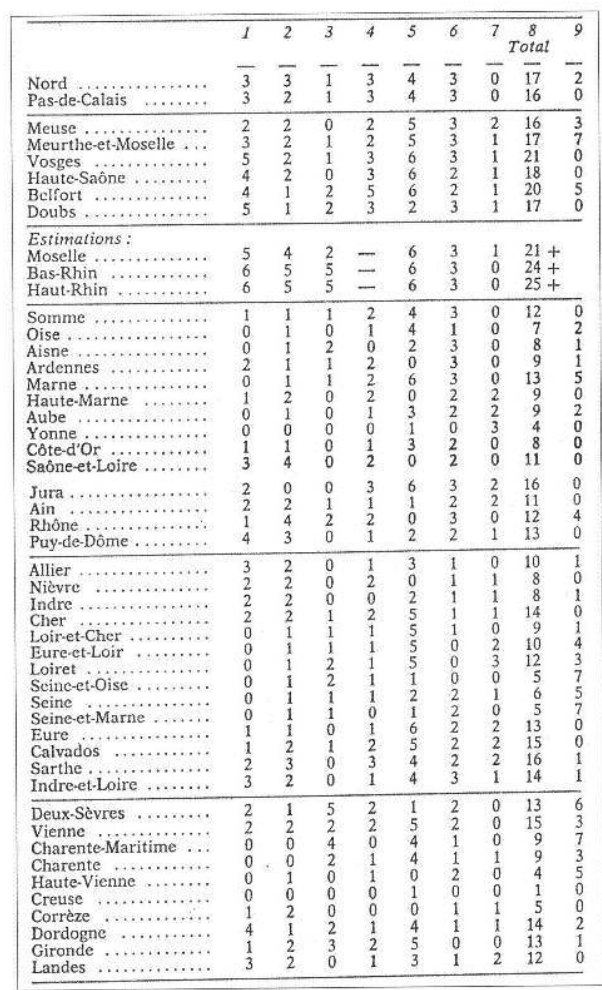




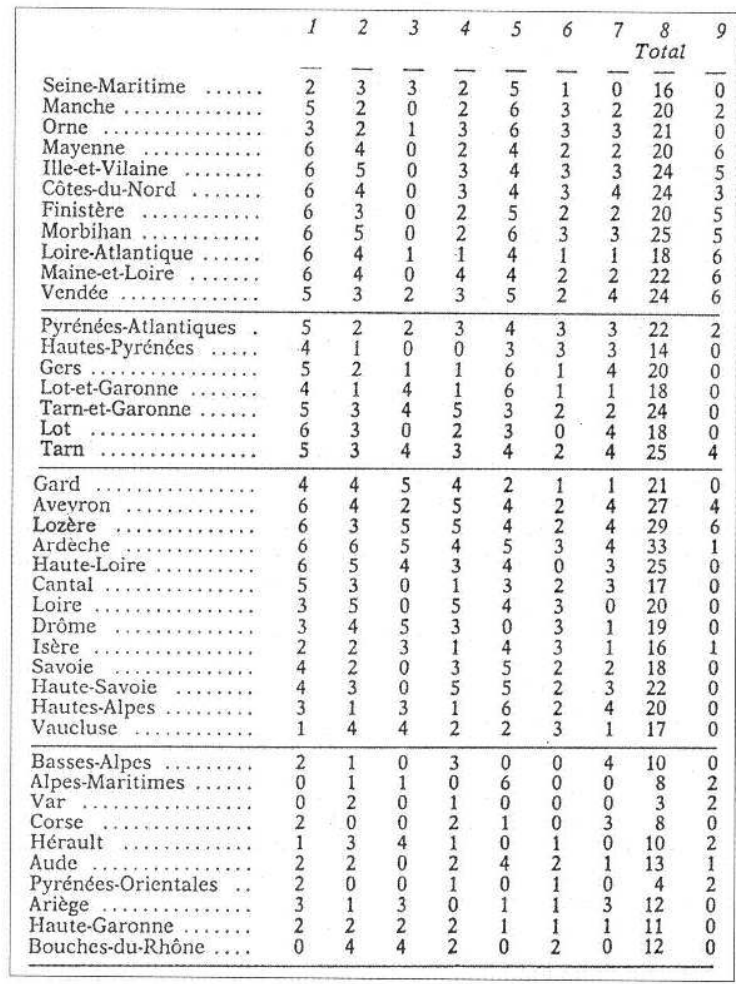

Un certain nombre de facteurs viennent contrarier les capacités initiales de résistance :

1. La plus ou moins forte implantation congréganiste antérieure n'était pas liée dans tous les cas à la ferveur des populations, les cas extrêmes étant la Saône-et-Loire, peu fervente mais comptant $50 \%$ des élèves chez les congréganistes; et, en sens contraire, le Doubs qui ne comptait que $27,4 \%$ d'élèves confiés à des congréganistes. Pour les garçons le lien est étroit avec la densité urbaine, les Frères des Écoles Chrétiennes étant souvent instituteurs communaux. La laïcisation a frappé durement dans certains cas : 22700 élèves perdus dans la Seine-Maritime, 10900 dans la Loire, 7200 dans le Pas-de-Calais. Inversement, la chute a été endiguée dans le Nord (98 046 élèves contre 100 725), la Meurthe-et-Moselle ou Belfort.

2. De nombreux évêques ont redouté la faiblesse des ressources matérielles nécessaires à l'ouverture d'écoles libres alors que la conjecture économique n'est pas bonne. Ainsi la forte pratique religieuse n'a pas empêché la chute brutale dans la Manche ou dans l'Orne ; et, plus encore, dans tout un ensemble de diocèses du Midi : Bayonne, Tarbes, Auch, Montauban, Cahors, Nîmes, Viviers, Le Puy, Saint-Flour, les diocèses de Savoie, Gap. Là, l'implantation congréganiste était forte au départ. Il n'a pas été possible d'ouvrir autant d'écoles libres. Inversement, il faut souligner le colossal effort que durent entreprendre des diocèses pauvres pour maintenir le réseau congréganiste (Albi, Rodez) et même pour l'accroître comme ce fut le cas à Mende où, il est vrai, le réseau était moins dense au départ.

3. La contribution des notables à l'effort entrepris se présente de diverses manières. Elle est déterminante quand le peuple est médiocrement attaché à l'Église: Seine, Seine-et-Oise, Seine-et-Marne, Eure-et-Loir. N'oublions pas que, pour les garçons surtout, il fallait ici en même temps offrir la gratuité pour décider des familles. Ce rôle des notables est l'une des raisons qui contribue, semble-t-il, à freiner le recul à Reims, Bordeaux, Toulouse, Montpellier, Toulon (sic) et Nice. Associé à un soutien populaire plus déterminé, cet appui rend compte des positions tenues dans le Nord et le Rhône, ou conquises à Nancy.

L'appui des notables reste un facteur essentiel lorsque le peuple est fidèle mais pauvre. Ainsi 
dans l'Ouest, sans préjudice des pressions exercées pour imposer le choix de l'école à ses métayers d'un côté ; et, en sens inverse, aux familles de fonctionnaires ou de la clientèle républicaine. Ce soutien des notables a manqué dans les chrétientés du Massif Central, des Alpes et des Pyrénées où peu nombreuses étaient les familles aisées. Inversement, des notables hostiles réduisent la défense scolaire à demeurer marginale (Côte d'Or, Calvados) ou à prendre d'autres formes que celle, coûteuse, du réseau congréganiste : les patronages paroissiaux ont été une réponse à la laïcisation de l'école publique. Ce n'est pas un hasard si les deux prêtres ruraux les plus précocement actifs à les prôner se trouvent l'un dans l'Aude, l'autre dans la Marne, les abbé Combe et Lecomte9.

4. Dans la Haute-Vienne, le gain de quelques 750 élèves est redevable à l'effort concurrent, mais inégal, que mènent les municipalités et l'Église pour combler le retard dans la scolarisation. C'est l'école au hameau qui achève de scolariser tous les enfants en Limousin comme dans l'Ouest et le Midi, et ce sont les effectifs de cette école qui rendent souvent compte des progrès de l'enseignement public.

5. Un autre facteur ne doit pas être sous-estimé : la fidélité des instituteurs publics à l'Église. Plusieurs évêques ont fait appel à eux dès 1882. Dans certaines chrétientés, telles que le Pays basque, le sud-est du Massif Central ou la Franche-Comté, instituteurs et institutrices sont et resteront longtemps des catholiques pratiquants : en 1909, c'est encore le cas pour $60 \%$ d'entre eux en Lozère. La moitié des curés interrogés (49 sur 98) qualifient la pratique religieuse des maîtres de «bonne » ou «très bonne », 29 de «nulle ». Dans ce dernier cas, il s'agit souvent des incidences du conflit entre les deux écoles. Ainsi, à Badaroux, «les institutrices ont cessé toute pratique depuis l'ouverture de l'école libre des filles ${ }^{10}$. Le 27 juin 1880 meurt, à Besançon, sœur Judith Guye, de la congrégation de la Charité de Besançon, et directrice durant 37 années de l'École normale de filles. L'année précédente, le recteur de l'Académie notait dans son rapport que « les jeunes filles qui sortent d'un pareil établissement n'ont de séculières que le nom » (8 août 1879). Chaque été les directrices participent à une retraite religieuse autour de sœur Judith. Mais, au dire de l'Inspecteur général, la même année "Il n'y a pas de meilleure École normale de filles en France ». A cette époque il y avait aussi, dans ce département, 272 sœurs enseignant dans les écoles publiques. Les institutrices formées par sœur Judith sont restées en fonction au moins jusqu'à la Grande-Guerre. Il ne faut donc pas s'étonner si, dans le Doubs, comme en quelques autres départements, et à la différence de la Seine, le crucifix est resté au mur de la classe bien après la loi de laïcisation ${ }^{11}$. C'est la forte pratique religieuse des institutrices laïques qui rendrait compte du développement relativement faible de l'école privée. Les prières continuent d'être dites en classe après 1882. «Je pourrais citer une foule d'instituteurs qui [les] font encore $»^{12}$. Bien que radical, le conseil municipal de Jougne adopte en 1897 un texte demandant « avec insistance le maintien des sœurs qui sont [ici] depuis 80 ans. Elles ont... élevé nos mères, nos épouses et nos enfants, elles ont la sympathie universelle des familles » 13. "Jusqu'ici, malgré les lois scolaires, plusieurs instituteurs et institutrices continuent à faire la prière à l'école en dehors des heures réglementaires de classe. Cet usage est toléré et même recommandé par leurs chefs hiérarchiques dans certains cas $\aleph^{14}$. On pourrait s'étonner, si l'on oubliait qu'il s'agit par ce moyen d'empêcher l'ouverture éventuelle d'une école libre. En 1893, les institutrices de Pontarlier conduisent leurs élèves à la procession de la Fête Dieu. «Si la directrice s'opposait à cette habitude », dit le rapport administratif, « elle porterait atteinte à son école... elle n'a répondu qu'aux sentiments des habitants qui quoique républicains sont catholiques". Dernière phrase qui résume bien la situation dans un département où le problème politique se posait avec moins d'acuité que dans les régions où le royalisme était solidement implanté. Des instituteurs tiennent à conserver les fonctions de chantre et d'organiste dont ils retirent au surplus du prestige et des avantages matériels, «c'est pour moi une perte de $200 \mathrm{~F}$ » écrit, en 1893, l'instituteur de Noël-Cerneux, un village de la montagne. Il supplie le préfet de l'autoriser à continuer ${ }^{15}$. A Montbenoit, et pour cinq communes, en 1898, dérogation est accordée d'utiliser les locaux scolaires pour le 
catéchisme «[...] ces communes commencent à se rallier au gouvernement de la République ». Elles appartiennent en effet à un petit pays où le souvenir de la résistance armée à la Révolution est resté très fort, à la différence du reste de la Franche-Comté. En 1901, le sénateur (de l'Ariège) Delpech, lit une lettre d'un de ses correspondants du Doubs qui pourrait citer " 200 écoles de notre département où, chose inouïe après 18 ans de laïcisation, on fait encore les prières en classe, on apprend le catéchisme et où le maître et la maîtresse vont surveiller leurs élèves à la messe et aux vêpres ». Par crainte d'une subversion sociale, le département évoluait alors vers cette droite modérée, mais républicaine, qui va caractériser une partie de la France de l'Est: en 1913, le marquis de Moustier était élu président du Conseil général.

6. Il est enfin des diocèses pauvres, religieux, mais non cléricaux et ne disposant ni de structures solides ni de tradition de résistance religieuse. A Digne, Perpignan, Pamiers comme à Ajaccio... le choc de la laïcisation amorce l'effondrement qui suivra le choc de la Séparation et donc de la suppression du budget des cultes.

21 Les interventions épiscopales ont pu infléchir, dans un sens ou un autre, le cours des événements : 60 lettres pastorales ont été publiées au moment des lois Ferry. Les évêques réagissent-ils différemment selon le peuple à la tête duquel ils ont été placés ?

Les interventions marquantes furent aussi nombreuses dans les deux cas, une vingtaine, donc en proportion plus fréquentes et de ton plus véhément là où l'appui populaire était assuré. Des évêques durent même freiner la politique de résistance absolue que préconisaient des états-majors de notables laïcs, ainsi dans le Nord. La nature des prises de position paraît avoir eu une influence sur le devenir de l'école libre. La grande activité du comité diocésain de Cambrai ${ }^{16}$, où $\mathrm{M}^{\mathrm{gr}}$ Duquesnay avait considéré la loi comme un "désastre pire que Sedan » $(1882)^{17}$ contraste avec l'attitude des archevêques de Rouen donnant une interprétation apaisante de la loi. Si la neutralité était observée, rien n'empêchait les catholiques d'envoyer leurs enfants à l'école publique. A Besançon, le modéré Paulinier, tout en protestant, compte surtout sur "l'intelligence du peuple franccomtois", et fait appel aux parents et aux catéchistes, auprès desquels il était sûr de trouver un écho.

Les évêques de l'Ouest, poussés ou non par leur clergé, les notables et les populations, se rangent parmi les intransigeants. Dès 1878, Saint-Brieuc et Quimper donnent une grande publicité à leurs protestations devant les premières laïcisations. En 1882, Séez parle "d'influence satanique ». Rennes, très violent de ton après l'expulsion des frères, fait de l'ouverture des écoles libres une œuvre vitale (1882). A Angers, $\mathrm{M}^{\mathrm{gr}}$ Freppel, qui était aussi député, aurait souhaité une déclaration collective de l'épiscopat, ce dont le dissuada l'archevêque de Paris. Il affirma le devoir strict des parents de préférer l'école congréganiste. De fait, la laïcisation changea finalement peu de choses dans le rapport des forces en présence ${ }^{18}$. En Loire-Atlantique, les masses paysannes sont nettement opposées à la bourgeoisie bleue nantaise. $\mathrm{M}^{\mathrm{gr}}$ Lecoq $\mathrm{n}^{\prime}$ est pas un évêque de combat mais il ne peut échapper à l'influence du Comité catholique au sein duquel les notables monarchistes sont en majorité. A partir de 1882, et surtout de 1886, les nombreuses bénédictions d'écoles libres, ici comme ailleurs se font accompagnées du cantique "Nous voulons Dieu "

«Nous voulons Dieu dans nos écoles

Pour qu'on enseigne à tous nos fils

La loi divine et ses paroles

Sous le regard du Crucifix » 
Le diocèse de Nantes sera l'un de ceux qui compteront au début du $\mathrm{XX}^{\mathrm{e}}$ siècle le plus grand nombre de catéchistes volontaires : 725 en 1904. Mais c'est avant tout le soutien à l'école libre qui mobilisera les énergies. A la rentrée 1882, le préfet autorisa les maîtres à faire un quart d'heure de catéchisme par jour à la demande des familles. Prudentes furent beaucoup de municipalités dans la laïcisation des écoles de filles. En 1910, les religieuses tenaient encore l'école communale à Vallet ${ }^{19}$.

Si l'on met à part l'évêque d'Annecy qui diffusa à 10000 exemplaires une protestation élevée contre les manuels de morale mais qui, faute de ressources, ne put développer un réseau d'écoles libres, ce sont ensuite plusieurs évêques du Midi pratiquant qui déployèrent la plus grande activité. Si celui de Tarbes ne put que déplorer son défaut de ressources, Agen, Auch, Albi, Saint-Flour, Valence, Rodez, Mende, Le Puy, Viviers et Nîmes invitèrent leurs diocésains à un gros effort en faveur des écoles libres. Depuis sa «Bretagne du Midi » (l'expression est de Pie IX), $\mathrm{M}^{\mathrm{gr}}$ Bourret intervint plusieurs fois. " Toute neutralité est blâmable ", aussi l'évêque de Rodez lança-t-il, non sans qu'il soit fondé on le sait, un appel à tous les maîtres pour qu'ils donnent à leur enseignement «l'esprit catholique ». Dans ce diocèse ne manquaient pas les instituteurs publics qui avaient un fils prêtre. Le Conseil général de l'Aveyron parvint à retarder durant 10 ans l'ouverture d'une École normale de filles laïcisée. Plusieurs de ces évêques étaient « du pays »: Bourret était né dans l'Ardèche ; $\mathrm{M}^{\mathrm{gr}}$ Bonnet, l'évêque de Viviers qui fut surnommé plus tard «l'évêque des écoles " était lozérien; $\mathrm{M}^{\mathrm{gr}}$ Costes, l'évêque de Mende était né dans l'Aveyron; à Perpignan, $\mathrm{M}^{\mathrm{gr}}$ Caraguel était né dans le Tarn ; à Albi, $\mathrm{M}^{\mathrm{gr}}$ Ramadié était né à Béziers : dans ce diocèse, le clergé, très remuant, s'appuyait sur une partie importante de la population. Dans la ville ouvrière de Mazamet, en janvier 1883, une pétition fut revêtue de 3420 signatures en faveur du crucifix, des prières, du catéchisme, de l'Histoire Sainte... et du maintien des Frères à l'école publique. Le gouvernement avait dû, d'ailleurs, adresser une circulaire apaisante sur les « emblèmes religieux » dès le 2 novembre 1882. Elle précède la célèbre circulaire de Jules Ferry aux instituteurs le 17 novembre 1883. A Montpellier, $\mathrm{M}^{\mathrm{gr}}$ de Cabrières était né à Beaucaire.

Il publia de Courtes observationss'en tenant à une interprétation juridique de la loi. Il constatait qu'elle laissait "subsister encore... quelques lambeaux de liberté. Ce sont des restes d'indépendance dont peuvent et doivent profiter les pères de famille ». Il leur appartient de faire respecter le jour de vacance hebdomadaire : il faut craindre des empiétements avec la circulaire du Ministre de la Guerre qui fixe pour les exercices militaires la journée du jeudi. Mais il n'incitait pas au refus de la loi tel que le préconisait certains légitimistes et le journal L'Univers. Ce même souci de respect de la loi se retrouve sous la plume du plus modéré des évêques de la région, le Franc-Comtois Besson, à Nîmes. Celui-ci se préoccupe avant tout du catéchisme. "Vous n'avez pas accès à l'école», écrit-il à ses prêtres. "N'importe, votre école est partout où règne la liberté: c'est l'église, le presbytère, la maison amie, le grand chemin. Appelez les enfants» (3 mai 1882). Alors que la crise du mûrier et le phylloxera ruinaient une partie de l'économie gardoise, $\mathrm{M}^{\mathrm{gr}}$ Besson avait créé, dès 1878 , un Comité de l'enseignement, puis une Caisse de secours et un Sou des écolesavec une cotisation de 3 francs par an. Il put ouvrir 120 écoles et répartir 100000 francs chaque année. $\mathrm{A}$ Viviers, $\mathrm{M}^{\mathrm{gr}}$ Bonnet parvint à maintenir $\mathrm{l}^{\prime}$ Ardèche dans le groupe des 9 diocèses de France comptant $47 \%$ et plus d'enfants scolarisés par les congréganistes :

\begin{tabular}{|c|c|c|c|c|}
\hline e & $47,7 \%$ & Aveyron & $48,9 \%$ & Loire-Atlantique \\
\hline & 50 & Mayenne & 51,4 & Maine-et-Loire \\
\hline he & 52,5 & Morbihan & 53,5 & Ille-et-Vilaine \\
\hline
\end{tabular}



Poitiers, à Marseille, Carcassonne, Toulouse où l'archevêque, malgré l'appui des légitimistes, semble se faire peu d'illusions sur l'esprit des pères de famille qui lisent La Dépêche. De nombreux diocèses du Bassin Parisien, du Centre ou du Midi sont sans ressources, leurs évêques le disent, à Amiens comme à Digne. Évoquant les pays de mission, Beauvais fait appel aux catéchistes volontaires, tout comme Clermont. Moins mal loti, l'archevêque de Reims fait un devoir de conscience aux parents d'envoyer leurs enfants aux écoles chrétiennes qui, de fait, connurent une légère progression d'effectifs. La recherche d'accommodation n'est pas sans laisser paraître une crainte pour l'avenir. A Bayeux, à Limoges comme à Montpellier, les évêques préconisent d'user de tous les moyens légaux pour obtenir que le jeudi soit respecté. Dans son appel aux parents à Châlons, $\mathrm{M}^{\mathrm{gr}}$ Meignan dit sa profonde inquiétude devant « ce fait nouveau dans notre histoire de l'indifférence légale et de la neutralité systématique du gouvernement quant à la religion des nouvelles générations ». On retrouve des expressions semblables à Limoges, Tulle, Autun ou Moulins. Dans ces diocèses, la crainte n'était pas imaginaire de voir des instituteurs manifester collectivement leurs sentiments vis-à-vis des déclarations épiscopales. Cela se produisit à Carcassonne où le nouvel évêque, $\mathrm{M}^{\mathrm{gr}}$ Billard, s'était lancé dans une polémique que dénoncèrent les députés Marcou et Omer Sarraut. L'évêque, qui était normand, avait été surpris par l'âpreté des conflits politico-religieux. Il faut dire qu'en Narbonnais, dans les Corbières, l'anticléricalisme était antérieur à 1815 et que l'évolution ultérieure l'avait renforcéé. Aussi avait-il invité les parents à éloigner leurs enfants des "écoles anticatholiques... comme d'une atmosphère malsaine où l'on respire la mort ». A Limoges, par contre, $\mathrm{M}^{\mathrm{gr}}$ Lamazou ne pouvait que souhaiter une entente sur le terrain de la neutralité. Celle-ci, insuffisante aux uns, intolérable aux autres, semblait compromise par les incidents survenus dans des départements dominés par un anticléricalisme masculin militant.

Toutefois l'épiscopat français, conscient de l'état de l'opinion publique, ne s'engagea pas dans l'attitude de refus qui avait été celle des catholiques belges. Comme l'écrivait l'archevêque de Paris au pape Léon XIII, le 9 juin 1882, "Malheureusement dans les trois quarts des communes de notre pays, il est impossible de créer et d'entretenir une seconde école ».

La stratégie adoptée de plus en plus fut celle de la défense religieuse, laquelle impliquait un repli sur les œuvres confessionnelles. Là où c'était possible cette défense s'incarna dans la mise en place et le soutien d'un réseau d'écoles libres. Un moyen plus souple, moins conflictuel, moins onéreux et qui, finalement, se révélera efficace, consista à multiplier les patronages paroissiaux, même en milieu rural. Cette stratégie suscita une réelle inquiétude dans les milieux laïques : "Je crains plus 10 patronages que 100 écoles" déclare Léon Bourgeois au Congrès de la Ligue de l'enseignement à Nantes, en $1894^{22}$. De même la mise en place, à Paris d'abord (1882), d'un réseau de catéchistes volontaires (le plus souvent des femmes et des jeunes filles) entend répondre à la charge nouvelle d'enseignement imposée au clergé, l'instituteur ne faisant plus apprendre le catéchisme. L'ouverture des salles d'asile (maternelles) correspond au même souci. Comme le patronage réunissait les enfants durant de longues heures le jeudi et le dimanche, on ne sous-estimera pas son influence dans la transmission du contenu de la foi. 

«l'esprit du pays ». Les instituteurs le savaient bien qui étaient, eux aussi « du pays »et prenaient en considération les vœux des familles. On sait par ailleurs que la première génération des instituteurs sécularisés était spiritualiste. On sait aussi quelles espérances les familles de la France rurale et paysanne placent alors dans l'école, l'un des vecteurs de la promotion sociale. On sait enfin qu'entre la morale laïque et la morale chrétienne les différences sont alors ténues, les bases de la première reposant sur les fondements de la seconde. Une situation qui va perdurer jusqu'à l'orée des années 1960.

Fin du XIXe siècle. Lithographie de Villain.

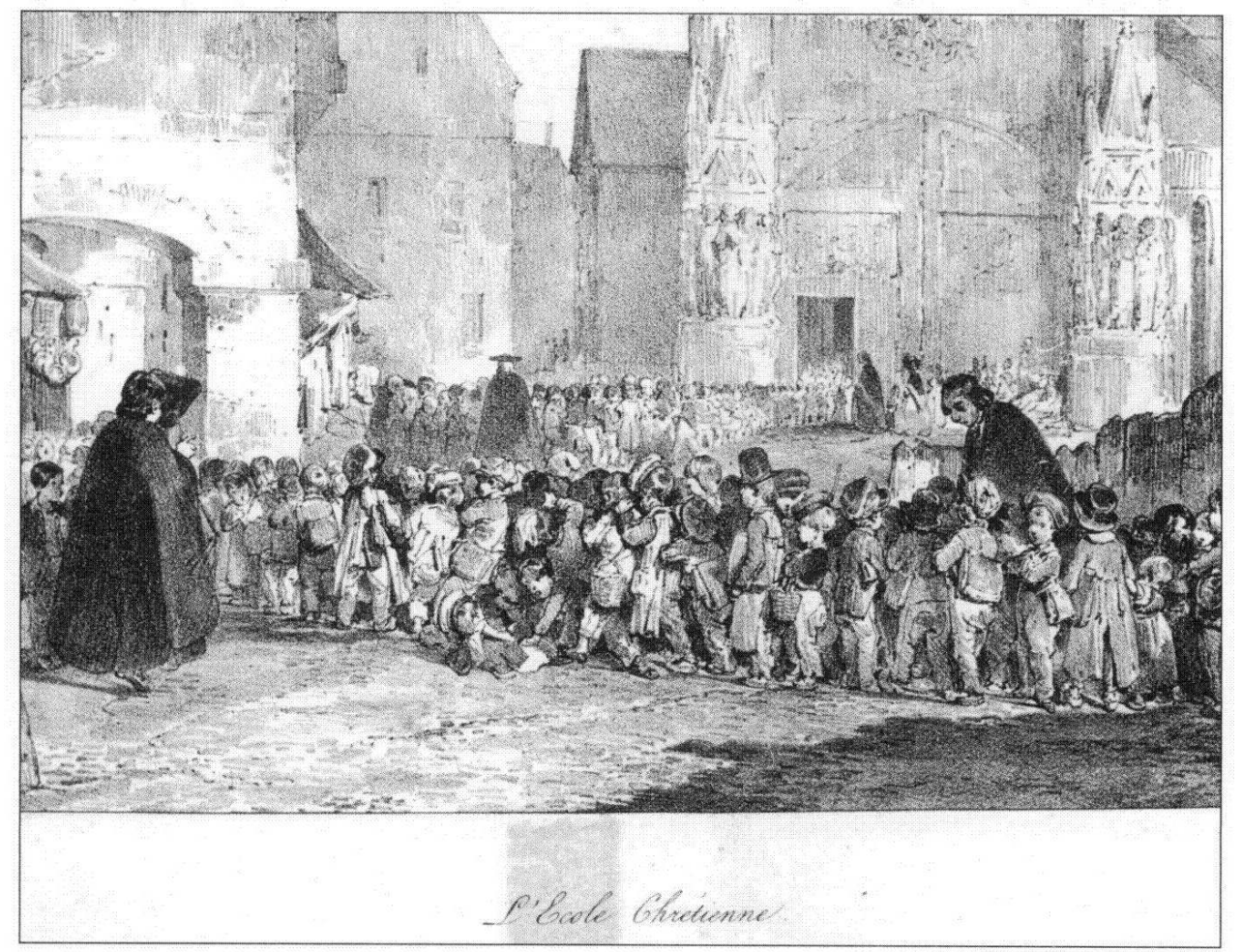

\section{NOTES}

1. Lettres de Jules Ferry, 1846-1898, Paris, 1914. LEGRAND L., L'influence du positivisme dans l'œuvre scolaire de Jules Ferry. Les origines de la laïcité, Paris, 1961.

2. Un résumé donnant l'essentiel dans: CHOLVY G. et HILAIRE Y.-M., «Chapitre 1: L'école publique devient laïque », in Histoire religieuse de la France contemporaine, T. 2, 1880-1930, Toulouse, 2 e édit. 1990.

3. A partir de la documentation statistique rassemblée dans les: Matériaux pour l'histoire religieuse du peuple français, XIX ${ }^{e}$-XXe siècle, Éditions du C.N.R.S., EHESS, FNSP, T. 1 (sous la dir. de BOULARD F.), 1982 ; T. 2 (sous la dir. de HILAIRE Y.-M.), 1987 ; T. 3 (sous la dir. de CHOLVY G.), 1992 ; T. 4, à paraître, (sous la dir. de DELPAL B.). 
4. Janvier 1883, cit. par GADILLE J., in La pensée et l'action politique des évêques français au début de la III République, 1870-1883, Hachette, 1967, T. II, Chap. IV, p. 195 sq.

5. Vocabulaire des proclamations électorales de 1881, 1885 et 1889, 1974.

6. MAYEUR J.-M., Cent ans d'esprit républicain. Histoire du peuple français, T. V, 1965, p. 209. Les droites n'ont eu que 90 élus contre plus de 200 en octobre 1877.

7. André Siegfried retenait seulement les filles. Il a semblé préférable de compter ensemble garçons et filles afin d'atténuer l'influence d'un trait de mentalité alors répandu, les filles étant beaucoup plus volontiers confiées à des maîtresses congréganistes que les garçons.

8. État par département, etc., annexe au rapport Jules Simon au Sénat le 8 décembre 1879.

9. CHOLVY G. «Patronages et œuvres de jeunesse dans la France contemporaine », in REHF, T. LXVIII, 1982, p. 235-256.

10. CAUSSE C. et TUFFERY A., Le diocèse de Mende au début du XX $X^{e}$ siècle, Mémoire de maîtrise, Montpellier, 1972. CHOLVY G., "Une chrétienté au XIX siècle: la Lozère, Cévennes et Gévaudan ", in Bulletin de la Société des Lettres, Sciences et Arts de la Lozère, N 18-19, 1972-1973, 1974, p. 264-382.

11. Et, dans certains cas, après la Seconde Guerre mondiale. Cf. PINARD J., « L'application des lois laïques dans le Doubs (1880-1905) ", in Mémoire de la Société d'émulation du Doubs, N²4, 1982, p. 14-32.

12. Le Petit Comtois, 3 janvier 1887.

13. Le 19 septembre 1897.

14. La Croix. Baume-les-Dames, 13 mai 1894.

15. L'article 125 de la loi de 1886 interdisait ces fonctions à l'instituteur. Il suscita un mécontentement qui n'est pas propre au département du Doubs.

16. Il n'existe pas encore d'évêché à Lille.

17. MÉNAGER B., La laïcisation des écoles communales dans le département du Nord (1879-1889). Lille, 1971.

18. RENOU A., La question scolaire dans le Maine-et-Loire de 1879 à 1891, Mémoire de maîtrise, Nantes, 1972.

19. LAUNAY M., «Les catholiques nantais et les lois scolaires 1880-1890 », in Actes de colloque: Colloque Freppel (Angers 1992), Letouzey, 1995, p. 67-77.

20. CHOLVY G., « Le devenir de l'école », in Histoire du Vivarais, Privat, 1988, p. 240 sq.

21. CHOLVY G., «Indifférence religieuse et anticléricalisme à Narbonne et en Narbonnais au XIX siècle ", in Actes de congrès: XLV Congrès de la Fédération historique du Languedoc méditerranéen et du Roussillon (Narbonne, 1972), Montpellier, 1973, p. 73-93.

22. Une émulation en résulta mais les instituteurs, dans leur ensemble, plaçaient leur confiance dans l'école. Il ne fut pas facile de recruter des normaliens et des instituteurs pour les mettre au service des œuvres péri ou post-scolaires. Les patronages laïques, qui souvent, ne furent que des garderies, en sont l'exemple, le scoutisme en est un autre. En 1900, on recensera 4168 patronages catholiques, 90 protestants et environ 1500 laïques ; en 1914, bien davantage, Cf. CHOLVY G. (s.d. de), Le Patronage ghetto ou vivier?, Nouvelle Cité, 1988. En 1898, au congrès de la Ligue de l'enseignement, Édouard Petit, recense 809 patronages scolaires : Nord, 132 ; Pas-de-Calais, 77 ; Seine. 88 ; Aude, 65 ; Seine-Maritime, 44 ; Gironde, 41 ; Marne, 39. Cette répartition montre qu'il s'agit bien d'une réponse à l'activité des patronages catholiques, chacun de ces départements correspondant à l'orientation nette des efforts pastoraux dans cette direction, y compris l'Aude et la Marne où sont actifs les deux prêtres pionniers du patronage paroissial, les abbés Combes et Leconte. 
INDEX

Mots-clés : anticléricalisme, école primaire, église, enseignement, France, IIIe république

Keywords : anti-clergy ideology, church, primary school, teaching, third republic

\section{AUTEUR \\ GÉRARD CHOLVY}

Université Paul Valéry de Montpellier 